\title{
Erratum: Functionally defined therapeutic targets in diffuse intrinsic pontine glioma
}

Catherine S Grasso, Yujie Tang, Nathalene Truffaux, Noah E Berlow, Lining Liu, Marie-Anne Debily, Michael J Quist, Lara E Davis, Elaine C Huang, Pamelyn J Woo, Anitha Ponnuswami, Spenser Chen, Tessa B Johung, Wenchao Sun, Mari Kogiso, Yuchen Du, Lin Qi, Yulun Huang, Marianne Hütt-Cabezas, Katherine E Warren, Ludivine Le Dret, Paul S Meltzer, Hua Mao, Martha Quezado, Dannis G van Vuurden, Jinu Abraham, Maryam Fouladi, Matthew N Svalina, Nicholas Wang, Cynthia Hawkins, Javad Nazarian, Marta M Alonso, Eric H Raabe, Esther Hulleman, Paul T Spellman, Xiao-Nan Li, Charles Keller, Ranadip Pal, Jacques Grill \& Michelle Monje

Nat. Med. 21, 555-559 (2015); doi:10.1038/nm.3855; published online 4 May 2015; corrected after print 15 June 2015

In the version of this article initially published online, there were four typographical errors in the manuscript text. The errors have been corrected in the PDF and HTML versions of this article.

\section{Erratum: Clonal evolution and resistance to EGFR blockade in the blood of colorectal cancer patients}

Giulia Siravegna, Benedetta Mussolin, Michela Buscarino, Giorgio Corti, Andrea Cassingena, Giovanni Crisafulli, Agostino Ponzetti, Chiara Cremolini, Alessio Amatu, Calogero Lauricella, Simona Lamba, Sebastijan Hobor, Antonio Avallone, Emanuele Valtorta, Giuseppe Rospo, Enzo Medico, Valentina Motta, Carlotta Antoniotti, Fabiana Tatangelo, Beatriz Bellosillo, Silvio Veronese, Alfredo Budillon, Clara Montagut, Patrizia Racca, Silvia Marsoni, Alfredo Falcone, Ryan B Corcoran, Federica Di Nicolantonio, Fotios Loupakis, Salvatore Siena, Andrea Sartore-Bianchi \& Alberto Bardelli

Nat. Med.; doi:10.1038/nm.3870; corrected online 26 June 2015

In the version of this article initially published online, Alberto Bardelli’s e-mail address was incorrect. The correct address is alberto.bardelli@unito.it. The error has been corrected in the print, PDF and HTML versions of this article. 